\title{
Correction to: Effects of mechanical property parameters on wrinkling behavior of thin-walled tubes in hydroforming process
}

\author{
Xiao-Lei Cui $^{1,2} \cdot$ Xiao-Song Wang $^{2} \cdot$ S. J. Yuan ${ }^{2}$
}

Published online: 1 December 2018

(C) Springer-Verlag London Ltd., part of Springer Nature 2018

\section{Correction to: Int J Adv Manuf Technol}

https://doi.org/10.1007/s00170-018-2706-2

In the original version of this article, the formula found in Section 2.3 and Eq. 1 were presented incorrectly.

The correct formula in Section 2.3 is shown below:

$$
\begin{aligned}
\bar{\sigma} & =\left\{\left[\left(\sigma_{1}-\sigma_{2}\right)^{2}+\left(\sigma_{2}-\sigma_{3}\right)^{2}+\left(\sigma_{3}-\sigma_{1}\right)^{2}\right] / 2\right\}^{1 / 2} \\
& =\left\{\left[\left(\sigma_{x}-\sigma_{y}\right)^{2}+\left(\sigma_{y}-\sigma_{z}\right)^{2}+\left(\sigma_{z}-\sigma_{x}\right)^{2}+6\left(\tau_{x y}^{2}+\tau_{y z}^{2}+\tau_{z x}^{2}\right)\right] / 2\right\}^{1 / 2}
\end{aligned}
$$

The correct formula in Eq. 1 is shown below:

$$
\sigma_{s} / \sigma_{f}=\frac{\sigma_{s}}{\sigma_{s}+\Delta \sigma}=\frac{1}{1+\frac{\Delta \sigma}{\sigma_{s}}}
$$

The original article has been corrected.

Publisher's Note Springer Nature remains neutral with regard to jurisdictional claims in published maps and institutional affiliations.

The online version of the original article can be found at https://doi.org/ 10.1007/s00170-018-2706-2

Xiao-Lei Cui

cuixiaolei2005@163.com; cuixiaolei@tyut.edu.cn

1 College of Materials Science and Engineering, Taiyuan University of Technology, Taiyuan 030024, People's Republic of China

2 National Key Laboratory for Precision Hot Processing of Metals, Harbin Institute of Technology, Harbin 150001, People's Republic of China 